\title{
Aspects Cliniques et Audiométriques des Nuisances Sonores dans les Centrales Electriques de la Ville de Ouagadougou
}

\author{
Oubian Souleymane, $M D$ \\ Chirurgien ORL, Centre Hospitalier Régional de Koudougou \\ Lankoandé Martin, MD \\ Anesthésiste Réanimateur, Centre Hospitalier Régional de Koudougou \\ Guetta Aboubacar, MD \\ Gyebré Yvette, PhD \\ Chirurgien ORL, Centre Hospitalier Universitaire Yalgado Ouédraogo \\ Seremé Moustapha, PhD \\ Chirurgien ORL, Centre Hospitalier Universitaire de Bogodogo \\ Ouoba Kampadilemba, PhD \\ Chirurgien ORL, Centre Hospitalier Universitaire Yalgado Ouédraogo, \\ University of Ouaga I Pr Joseph Ki-Zerbo, Burkina Faso
}

Doi:10.19044/esj.2020.v16n6p395 ～URL:http://dx.doi.org/10.19044/esj.2020.v16n6p395

\section{Résumé}

Introduction: Les nuisances sonores sont néfastes à la santé. Les surdités constituent une pathologie grandissante avec l'urbanisation, l'industrialisation du fait des nuisances sonores secondaires. Des mesures de protections doivent être mises en œuvre pour la sécurité des travailleurs et doivent être basées sur les risques potentiels. L'objectif de cette étude était d'évaluer la nuisance sonore et son impact sur la santé des travailleurs. Méthodes : Il s'est agi d'une étude prospective par enquête des travailleurs avec évaluation audiométrique menée dans les quatre centrales éléctriques de la Société Nationale d'Electricité du Burkina (SONABEL). L'étude a été menée du 19 juillet 2016 au 03 aout 2016. Ont été inclus les travailleurs desdites centrales en activité pendant la période d'étude. Une audiométrie tonale linéaire (ATL) a été réalisée après une analyse des caractéristiques sociodémographiques et professionnelles. L'analyse des données a été faite avec EPI info 3.5.1. Résultats: Nous avons noté $103(94,5 \%)$ hommes contre 6 femmes. L'âge médian était de 38 ans et 27,5\% occupait le poste d'agent de conduite. Des antécédents d'otites étaient observés dans 30,3\%. La sonométrie a noté une moyenne de 105,05 décibels $(\mathrm{db})$ dans les salles de production et $74,1 \mathrm{db}$ dans les salles de commandes. Une surdité était observée dans 16,5\%. 
Douze (40\%) des 30 agents de conduite avaient une anomalie d'audition. La durée moyenne d'exposition au bruit était de 11,24 ans. L'exposition était intermittente dans $80,7 \%$ et continue dans $19,27 \%$. Conclusion : Le niveau et la durée d'exposition sonore est élevé dans les centrales industrielles de la SONABEL dans la ville de Ouagadougou. Des mesures de protection doivent être promues pour une sécurité au travail.

Mots Clés : Nuisance, Sonore, SONABEL, Ouagadougou

\title{
Clinical and Audiometric Aspects of Sound Nuisance in Power Plants of the City of Ouagadougou
}

\author{
Oubian Souleymane, $\mathrm{MD}$ \\ Chirurgien ORL, Centre Hospitalier Régional de Koudougou \\ Lankoandé Martin, MD
}

Anesthésiste Réanimateur, Centre Hospitalier Régional de Koudougou

Guetta Aboubacar, MD

Gyebré Yvette, PhD

Chirurgien ORL, Centre Hospitalier Universitaire Yalgado Ouédraogo

Seremé Moustapha, PhD

Chirurgien ORL, Centre Hospitalier Universitaire de Bogodogo

Ouoba Kampadilemba, PhD

Chirurgien ORL, Centre Hospitalier Universitaire Yalgado Ouédraogo,

University of Ouaga I Pr Joseph Ki-Zerbo, Burkina Faso

Abstract

Introduction: Noise pollution is harmful to health. Deafness is a growing pathology with urbanization and industrialization due to secondary noise pollution. Protective measures must be implemented for the safety of workers and must be based on potential risks. The objective of this study is to evaluate the level of noise pollution and its impact on workers' health. Methods: This was a prospective survey study of workers with audiometric evaluation conducted in the four power stations of the National Society of Power of Burkina. The study was conducted from July 19, 2016 to August 3, 2016. It included the workers of the said power stations in operation during the study period. A linear tonal audiometry (LTA) was conducted after an analysis of socio-demographic and occupational characteristics. Data analysis 
was carried out with EPI info 3.5.1. Results: We noted 103 (94.5\%) males versus 6 females. The median age was 38 years and $27.5 \%$ held the position of driving officer. A history of ear infections was observed in $30.3 \%$. Sound level measurements averaged 105.05 decibels $(\mathrm{db})$ in the production rooms and $74.1 \mathrm{db}$ in the control rooms. Deafness was observed in $16.5 \%$. Twelve (40\%) of the 30 driving agents had a hearing abnormality. The average duration of exposure to noise was 11.24 years. Exposure was intermittent in $80.7 \%$ and continuous in $19.27 \%$. Conclusion:The level and duration of sound exposure is high in SONABEL's industrial plants in the city of Ouagadougou. Protective measures must be promoted for safety at work.

Keywords: Nuisance, Noise, SONABEL, Ouagadougou

\section{Introduction}

Le bruit est l'un des polluants les plus fréquents et les plus menaçants. Le nombre de personnes souffrantes de déficience auditive est en augmentation dans le monde. Si aucune mesure n'est prise, d'ici à 2030, près de 630 millions de personnes seront atteintes de déficience auditive incapacitante et, d'ici à 2050, ce nombre pourrait augmenter et atteindre 900 millions(Organization. 2018). Présent dans la quasi-totalité des secteurs d'activité économique, le bruit en milieu professionnel est en progression avec les progrès croissants des technologies et l'utilisation à outrance d'outillages et de machines sophistiqués. De ce fait, le bruit en milieu professionnel demeure à l'origine de manifestations cliniques diverses dont la plus fréquente est la surdité professionnelle. Cette pathologie demeure d'actualité malgré le développement des techniques de protection collective et la mise en œuvre de moyens de protection individuelle (M. Concha-Barrientos, D. CampbellLendrum, and K. Steenland 2004). Elle est grave par son caractère irréversible et par son évolution inéluctable vers le handicap avec toutes ses répercussions sanitaires, sociales et professionnelles. L'objectif de notre travail était d'évaluer le niveau de nuisance sonore et son imacpt sur la santé des professionnels.

\section{Méthodes}

Il s'est agi d'une étude prospective menée dans les quatre centrales de la Société Nationale d'Electricité du Burkina (SONABEL) que sont les centrales de Gounghin, de Kossodo, de Paspanga et de Komsilga. Les locaux des différentes centrales comprennent des salles de machines, de commande, des bureaux pour les travailleurs et des salles de dépotage. Le personnel se compose d'ouvriers qualifiés et d'ouvriers non qualifiés. L'étude a été faite du 19 juillet 2016 au 03 aout 2016. Un échantillonnage exhaustif a été fait. Ont été inclus tous les travailleurs desdites centrales en activité pendant la période 
d'étude. Tous les travailleurs ont subis un éxamen médical et des audiométries tonales linéaires (ATL) ont été réalisées. Les variables sociodémographiques, cliniques et audiométriques ont été étudiées. L'analyse des données a été réalisée grâce aux logiciels EPI info 3.5.1.

\section{Résultats}

Nous avons noté $103(94,5 \%)$ hommes contre 6 femmes Sex-ratio = 17,1). L'âge médian était de 38 ans. La répartition des patients par tranche d'âge est présentée sur le tableau I.

Tableau I : Répartition des travailleurs selon l'âge $(\mathrm{n}=109)$

\begin{tabular}{ccc}
\hline Tranche d'âge & Effectif & Proportion $(\%)$ \\
\hline$\leq 19$ & 1 & 0,9 \\
$20-29$ & 20 & 18,3 \\
$30-39$ & 43 & 39,5 \\
$40-49$ & 28 & 25,7 \\
$50-59$ & 17 & 15,6 \\
Total & 109 & 100 \\
\hline
\end{tabular}

Trente sur 109 personnes $(27,5 \%)$ occupaient le poste d'agent de conduite comme l'illustre la figure 1 ci-après

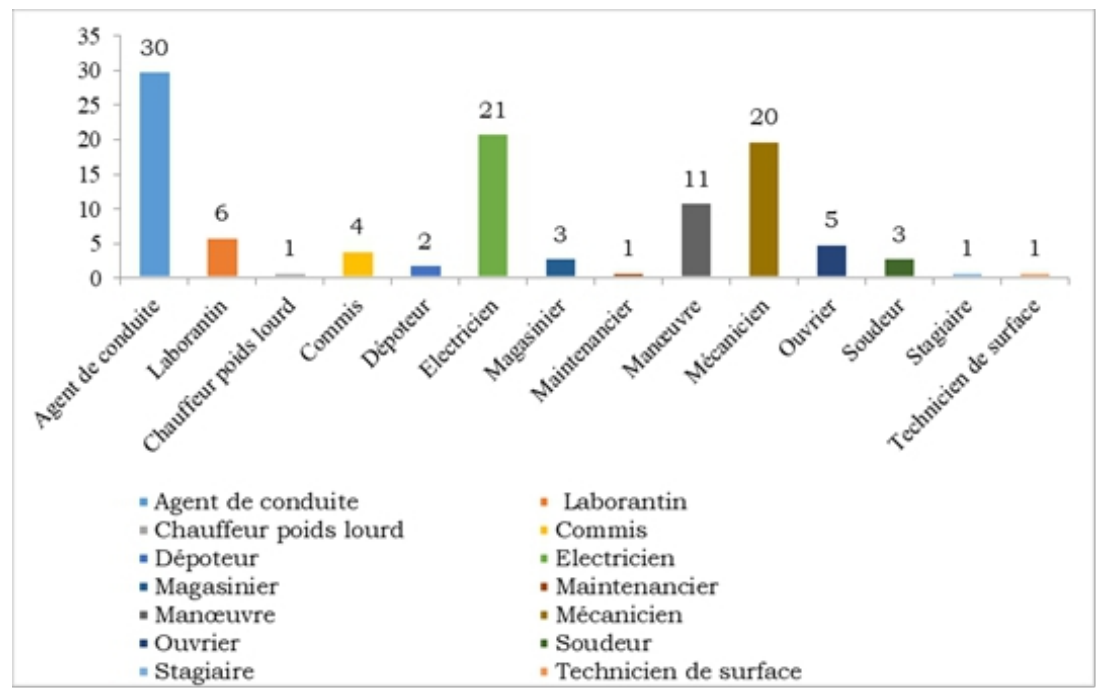

Figure 1 : Répartition des travailleurs selon l'activité $(\mathrm{n}=109)$

Des antécédents d'otites étaient observés chez 33 personnes selon le tableau II ci-après. 
Tableau II : Répartition selon les antécédents des travailleurs $(\mathrm{n}=33)$

\begin{tabular}{lllll}
\hline Types antécédents & Oui / Non & Nature & Effectif & Pourcentage \\
\hline $\begin{array}{l}\text { Antécédents } \\
\text { personnels }\end{array}$ & Non & & & \\
Médicaux & Oui & & 33 & 100 \\
& & Otites aigues & 27 & 81,8 \\
& & Otites chroniques & 6 & 18,2 \\
& & Rhinopharyngites & 4 & 12,1 \\
& & Sinusites & 26 & 78,8 \\
& & Méningite & 5 & 15,1 \\
& & Rougeole & 15 & 45,5 \\
& & Oreillons & 23 & 69,7 \\
& & Hémoglobinopathie & 2 & 6,0 \\
Chirurgicaux & Oui & Diabète & 1 & 3,0 \\
& & Lésion du tympan & 1 & 18,2 \\
& & Barotraumatisme & 1 & 3,9 \\
& & Traumatisme & 4 & 12,1 \\
Antécédents & Crânien & & \\
familiaux & & Surdité & 4 & 12,1 \\
\hline
\end{tabular}

Les agents travaillant en salle de production étaient majoritaires comme l'illustre la figure 2 .

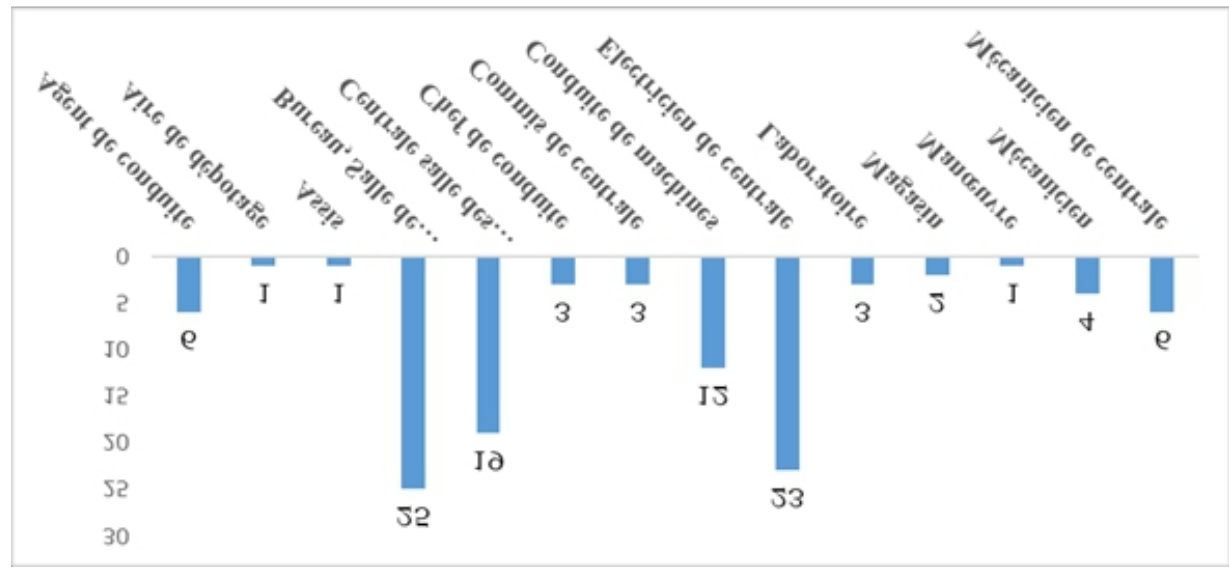

Figure 2: Répartition des travailleurs selon le poste de travail $(\mathrm{n}=109)$

L'examen clinique a révélé sept cas d'anomalies dont quatre cas $(3,67 \%)$ de bouchons de cérumens, un corps étranger à type de coton tige dans deux cas $(1,8 \%)$ et un cas d'otite externe (0,9\%). L'examen ORL complet était anormal chez 09 travailleurs avec une hypertrophie de cornet dans 03 cas et un cas de torus du palais osseux (tableau III). 
Tableau III : Répartition selon l'examen ORL ( $\mathrm{n}=9)$

\begin{tabular}{ll}
\hline Examen ORL complet & Effectif \\
\hline Hypertrophie de la cornée inférieure gauche & 1 \\
Excroissance osseuse au niveau du palais & 1 \\
HCI gauche + Hypertrophie des AP non destructives & 1 \\
Hypertrophie bilatérale des cornées & 3 \\
Hypertrophie cornée sup droite + Hypertrophie unilatérale amygdale gauche & 1 \\
Hypertrophie des cornées & 1 \\
Lésions prurigineuses vésiculeuse du pavillon bilatéral & 1 \\
Total & 9 \\
\hline
\end{tabular}

ORL : Otho-rhyno-laryngologie, HCI : Hypertrophie des Cornets Inférieurs AP : Amygdales Palatines

La sonométrie réalisée dans tous les sites a noté une valeur moyenne de 105,05 décibels (db) avec des extrêmes de 100db à 112,3db dans les salles de production. Le site de Komsilga a eu le niveau le plus élevé avec 112,3db. Dans les salles de commandes le niveau moyen était de $74,1 \mathrm{db}$ avec des extrêmes allant de 65,7db à Gounghin à 84,1db à Kossodo (figure 3).

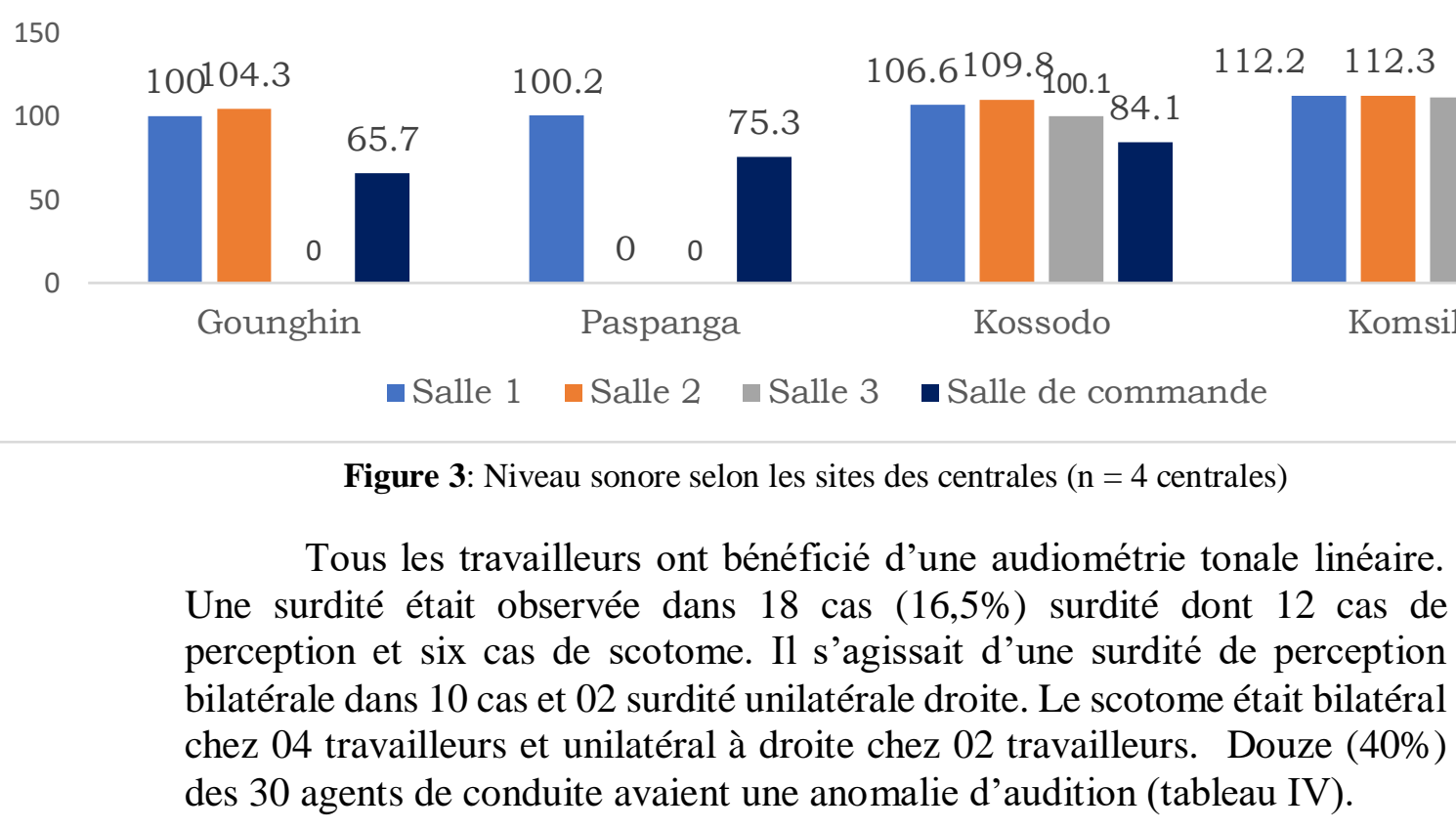


Tableau IV: Répartition des cas d'anomalies selon la profession $(\mathrm{n}=109)$

\begin{tabular}{llll}
\hline Profession & Surdité légère & Scotome & Total \\
\hline Agent de conduite & $\mathbf{8}$ & $\mathbf{4}$ & $\mathbf{1 2}$ \\
Ouvrier & $\mathbf{2}$ & $\mathbf{2}$ & $\mathbf{4}$ \\
Mécanicien & $\mathbf{2}$ & & $\mathbf{2}$ \\
Chauffeur poids lourd & 0 & 0 & 0 \\
Laborantin & 0 & 0 & 0 \\
Commis & 0 & 0 & 0 \\
Dépoteur & 0 & 0 & 0 \\
Electricien & 0 & 0 & 0 \\
Magasinier & 0 & 0 & 0 \\
Maintenancier & 0 & 0 & 0 \\
Euvrier & 0 & 0 & 0 \\
Soudeur & 0 & 0 & 0 \\
Stagiaire & 0 & 0 & 0 \\
Technicien de surface & 0 & 0 & 0 \\
Total & & & \\
\hline
\end{tabular}

Dans notre étude la durée moyenne d'exposition au bruit est de 11,24 ans avec des extrêmes de moins d' 1 an et 39 ans. La tranche de durée la plus d'exposition la plus touché par la surdité est 0 à 10 ans ( 5 cas surdité légère, 2 cas scotomes) suivie de la tranche de 11 à 20 ans (3 cas surdité, 2 cas scotomes). L'exposition était intermittente chez 88 (80,73\%) personnes continue chez $21(19,27 \%)$ personnes. Une baisse auditive était retrouvée chez 13 travailleurs en exposition continue contre 5 travailleurs en exposition intermittente (tableau V).

Tableau V : Durée et nature de l'exposition sonore des surdités $(n=18)$

\begin{tabular}{llll}
\hline Caractéristiques de l'exposition & Surdité légère & scotome & Effectif \\
\hline Durée de l'exposition (ans) & & & \\
\hline $0-10$ & 5 & 2 & 7 \\
$11-20$ & 3 & 2 & 5 \\
$21-30$ & 2 & 1 & 3 \\
$\geq 31$ & 2 & 1 & 3 \\
Total & $\mathbf{1 2}$ & $\mathbf{6}$ & $\mathbf{1 8}$ \\
Durée de l'exposition & & & \\
Continue & 8 & 5 & 13 \\
Intermittente & 4 & 1 & 5 \\
Effectif total & $\mathbf{1 2}$ & $\mathbf{6}$ & $\mathbf{1 8}$ \\
\hline
\end{tabular}

\section{Discussion}

Cette étude présente des limites liées à sa durée courte qui n'a pas permis d'examiner tous les travailleurs des sites industriels. Aussi le mode de fonctionnement des centrales qui ont des équipes de permanences du jour et 
de garde a influencé nos résultats. Malgré cela notre étude a pu relever des anomalies pouvant être prévenues.

Les travailleurs des centrales sont en majorité jeunes. Nos résultats sont comparables à ceux de Benzarti et coll en Tunisie (Benzarti Mezni et al. 2014) qui trouvaient un âge moyen de 37,1 ans. Nous pouvons expliquer cela par le fait qu'en Afrique et particulièrement au Burkina Faso les ouvriers sont jeunes.

Les femmes sont moins rencontrées dans les sociétés de production d'électricité malgré une population Burkinabè en majorité féminine. Amel Arib et coll (Mezdad, Mahamed, and Mahamed 2016) notait chez les travailleurs d'une industrie de l'électroménager en Algérie exclusivité masculine. Cette prédominance a également été rapportée par Kammoun et coll (Naceur et al. 2006) qui constate aussi que les hommes étaient plus représentés. Cette prédominance masculine pourrait s'expliquer par le fait qu'en Afrique, certaines professions sont encore réservées aux hommes (ouvriers industriels, soudeurs, électriciens, soudeurs...).

Toutes les catégories professionnelles étaient représentées dans notre étude avec une prédominance des agents de conduite $(27.5 \%)$ ainsi que les électriciens (19.2\%). Nos résultats corroborent avec ceux de Henchi et coll (Salah et al. 2008) qui trouvaient $29.9 \%$ d'agent de conduite et $15.2 \%$ d'électriciens. Cette situation pourrait s'expliquer par le fait que le besoin en agent de conduite et en électricien est les plus élevé dans les industries.

Des antécédents d'affections ORL étaient observés chez 30,3\%. Aucun patient n'avait un antécédent de surdité. Malgré la présence des antécédents ORL significatifs certains patients ont été embauchés. En Tunisie des patients présentant des antécédents otologiques ont eu plus de complications que ceux qui n'en avait pas(Salah et al. 2008). Cette situation s'explique soit du fait des simulacres de visite médicale d'embauche, ou d'une absence de politique de prévention des risques professionnels. La possibilité d'obtenir des examens objectifs suggèrent une mauvaise attention dans la sélection des travailleurs.

Une mesure du bruit était réalisée dans tous les sites dans notre étude. La valeur moyenne était de $105.05 \mathrm{db}$ dans les salles à machine des 04 sites contre $74.125 \mathrm{db}$ dans les salles de commandes Ndiaye et coll(Ndiaye et al. 2014) au Sénégal trouvaient une moyenne de $87.86 \mathrm{db}$. Wafid M et coll(Wafid Mohamed Benzian*, Rabia Medjane, Younes Ghezini n.d.) en Algérie trouvaient un niveau sonore moyen de 82 à $95 \mathrm{db}$. Le niveau sonore élevé dans notre étude pourrait s'expliquer par la qualité des machines utilisée à la SONABEL. Ces valeurs sont au-delà des limites recommandées(Authority 2010). La mise en œuvre des recommandations de prévention de l'OMS permettra de réduire le risque (Who 2011).

Dans notre étude la durée moyenne d'exposition au bruit est de 11,24 ans. Ndiaye et coll au Sénégal (Ndiaye et al. 2014) trouvaient un déficit auditif 
chez les travailleurs avec une durée d'exposition de 16 à 30 ans. Dans notre étude la tranche d'âge d'exposition la plus touchée est de 0 à 10 ans. Ceux-ci s'expliqueraient par le fait qu'à la SONABEL après un temps d'exposition les agents qui travaillaient en salle de machine sont remplacés par d'autres agents. L'exposition était intermittente dans $80,7 \%$ et continue dans $19,3 \%$. Une baisse auditive était retrouvée chez 13 travailleurs en exposition continue contre 5 travailleurs en exposition intermittente. L'exposition continue augmente le risque de morbidité auditive comparée à l'exposition intermittente(Michell 2007)(Strauss et al. 2012). Selon la littérature, l'exposition doit être limitée en intensité et en durée(M. Concha-Barrientos, D. Campbell-Lendrum, and K. Steenland 2004)(Who 2011)(Nelson et al. 2005).

Dans notre étude la surdité légère type perceptionnelle était élevée chez les travailleurs en exposition continue. Nos résultats corroborent avec ceux de Ladhari et coll(Ladhari et al. 2014) en Tunisie qui trouvaient un pourcentage élevé de surdité chez les travailleurs en exposition continue. Nos résultats corroborent avec ceux de Benzarti et coll en Tunisie(Benzarti Mezni et al. 2014) et de P. Forget (Forget 2011). La surdité de perception est due au faite que l'atteinte auditive rencontrée dans les industries est une atteinte neurologique. Dans notre étude tous les cas de surdités rencontrées étaient des surdités légères. Nos résultats sont différents de ceux de Ladhari et coll (Ladhari et al. 2014) qui trouvaient de surdités légère et de surdités moyennes. La surdité légère dans notre étude pourrait s'expliquer par l'application adéquate des mesures de protections chez les travailleurs de la SONABEL.

Dans notre étude 10 travailleurs avaient une surdité perceptionnelle bilatéral soit $83,3 \%$ et 02 travailleurs avaient une surdité unilatérale à droit dans $16.6 \%$. Le scotome était bilatéral chez 04 travailleurs et unilatéral droit chez 02 travailleurs. Nos résultats sont différents de Benzarti et coll (Benzarti Mezni et al. 2014)en Tunisie qui trouvaient 79.5\%. Par contre nos résultats sont comparable à ceux de Benzarti et coll(Benzarti Mezni et al. 2014) en Tunisie qui trouvaient une atteinte perceptionnelle bilatérale dans $85,1 \%$. Pour Pesseye et coll (Pessey, Deguine, and Wanna 2004) l'audiogramme doit toujours prendre en considération les fréquences sur les $3000 \mathrm{~Hz}$ et $6000 \mathrm{~Hz}$ car dans certains cas ce sont les seules fréquences atteintes. Nous pouvons dire que l'atteinte auditive est plutôt liée à une mauvaise utilisation des mesures de protections.

\section{Conclusion}

Les travailleurs de la SONABEL débutent leurs professions avec souvent des antécédents lésionels otologiques et son sont jeunes. Ils sont exposé à un niveau de nuisance sonore élevé. Les surdités professionnelles sont les complications de cette exposition nuisante. Une rigeur de la procédure 
de selections des candidats, des mesures de prévention et de protection sont nécessaires pour une méilleurs sécurité des travailleurs. Une étude sur les déterminants de la surdité permettrait d'identifier les facteurs de risque de de surditité professionnelle pour mieux prévenir.

\section{References:}

1. Authority, Health \& Safety. 2010. "Noise at Work: Guidance for Employers." : 1-13.

2. Benzarti Mezni, A. et al. 2014. "Profil Étiologique Des Surdités d'origine Professionnelle. À Propos de 67 Cas." Archives des Maladies Professionnelles et de l'Environnement 75(3): S21. https://linkinghub.elsevier.com/retrieve/pii/S1775878514001076.

3. Forget, P. 2011. 'Évaluation de La Nocivité Auditive Moyenne d' Une Exposition Professionnelle à Des Bruits Impulsifs §." : 16-19.

4. Ladhari, N. et al. 2014. "Profil Audiométrique et Prévalence Des Troubles Auditifs Chez 420 Téléconseillers." Archives des Maladies Professionnelles et de l'Environnement 75(3): S20. https://linkinghub.elsevier.com/retrieve/pii/S1775878514001040.

5. M. Concha-Barrientos, D. Campbell-Lendrum, and K. Steenland. 2004. "Occupational Noise: Assessing the Burden of Disease from Work Related Hearing Impairement at National and Local Levels." (9).http://www.inspq.qc.ca/pdf/publications/712BruitMilieuTravail.p df.

6. Mezdad, Amel Arib Ep, Amer Lamara Mahamed, and Amer Lamara Mahamed. 2016. "Évaluation Du Déficit Auditif Moyen Chez Les Travailleurs D’Une Industrie De L'Électroménager.” Archives des Maladies Professionnelles et de l'Environnement 77(3): 539. https://linkinghub.elsevier.com/retrieve/pii/S177587851630460X.

7. Michell, Karen. 2007. "Auditing Occupational Audiometric Testing a Pilot Study."

8. Naceur, H Kammoun B E N et al. 2006. "Reconnaissance et Réparation de La Surdité Professionnelle En Tunisie . À Propos d' Une Étude Épidémiologique Évaluation de 1' Exposition Professionnelle Au Bruit : Apports de La Norme Française En Milieu de Travail Non Bruyant ." : 31084.

9. Ndiaye, M. et al. 2014. "Évaluation Du Risque Bruit Au Niveau Du Site Acide Des Industries Chimiques Du Sénégal (ICS).” Archives des Maladies Professionnelles et de l'Environnement 75(3): S19. https://linkinghub.elsevier.com/retrieve/pii/S1775878514001015.

10. Nelson, DI, RY Nelson, M Concha-Barrientos, and M Fingerhut. 2005. "The Global Burden of Occupational Noise-Induced Hearing Loss." Am J Ind Med 48(6): 446-58. 
11. Organization., Wolrd Health;2018. 2018. "Souffrant de Déficience Auditive Est En Augmentation! Préparons -Nous." : 1-4. www.who.int/deafness/world-hearing-day/whd-2018/en.

12. Pessey, J. J., O. Deguine, and G. Wanna. 2004. "Pathologies Otorhinolaryngologiques Au Cours Des Explosions." EMC - OtoRhino-Laryngologie 1(3): 225-31.

13. Salah, H Haj, A Gaaliche, T Khalfallah, and M Akrout. 2008. " Se Auditive Dans Les Apport de La Prothe's Professionnelles Surdite." : 593-99.

14. Strauss, S et al. 2012. "Prevalence and Degree of Noise-Induced Hearing Loss in South African Gold Miners." Occupational Health Southern Africa 18(20-25): 20-25. http://repository.up.ac.za/bitstream/handle/2263/21979/Strauss_Preva lence(2012).pdf?sequence $=1$.

15. Wafid Mohamed Benzian*, Rabia Medjane, Younes Ghezini, Baghdad Rezk-Kallah. "Ame ' Nagement Des Conditions de Travail Du Personnel Fe ' Minin Roulant Des e' Tablissements Commerciaux Trains Au Cours de La Grossesse Profil Audiome' Trique Des Travailleurs d' Une Verrerie Industrielle." : 541.

16. Who. 2011. "Burden of Disease from Burden of Disease From." 